\title{
On-Detector Electronics for the ATLAS TileCal Demonstrator
}

\author{
S. Muschter, H. Aakerstedt, K. Anderson, C. Bohm, G. Drake, M. Oreglia, A. Paramonov and F. Tang \\ on behalf of the ATLAS Tile Calorimeter System
}

$: \mathbf{T}$ HE iron-scintillator ATLAS Tile calorimeter [2], [1] is partitioned into 4 cylindrical sections, each composed of 64 wedge shaped modules. The electronics are located in extractable "super-drawers" at the base of each wedge. This placement outside the calorimeter means that they are shielded by the calorimeter iron. The super-drawers contain 45 or 32 PMTs depending on section, with each calorimeter cell read out by two PMTs. In all there are close to 10000 PMTs reading out about 5000 calorimeter cells. The PMT signals are presently amplified and shaped by front-end ${ }_{1}$ boards (3-in-1) with high and low gains (gain ratio 64) 42 4 In this way it is possible to cover a dynamic range of $16_{3}$ 15 bits using two 10 bit ADCs. The signals are digitized and 6 stored in the digitizer board awaiting a corresponding leve $4_{5}$ 71 trigger accept before transmitting the data off the detector ${ }_{46}$ ${ }_{18}$ The trigger decision is formed from summed analog signal $\$_{7}$ from the front-end boards, representing tower geometries, and $\mathrm{d}_{8}$ sent to the level 1 trigger in the counting room outside the detector. The triggered readout leads to a significant reductiono of the required bandwidth and cost. This was essential when designing the original system.

By 2023 the LHC accelerator will be upgraded to an ${ }^{5}$ instantaneous luminosity increased by a factor of 5 and the average luminosity increased by a factor of 10 . This ${ }^{7}$ means more complex events and increased radiation exposures to the electronics. Furthermore, since the initial TileCat9 design there have been significant technology developments $0^{0}$ Large bandwidths are less costly and radiation tolerance in ${ }^{1}$ integrated circuits more easily achieved. In the upgraded 2 design intended for 2023 all data are read out to the counting 3 room, allowing the first level trigger data to be formed outside the detector. This provides more detailed digital data to the initial trigger, allowing more sophisticated trigger algorithms to compensate for the more complex backgrounds expected at high luminosity.

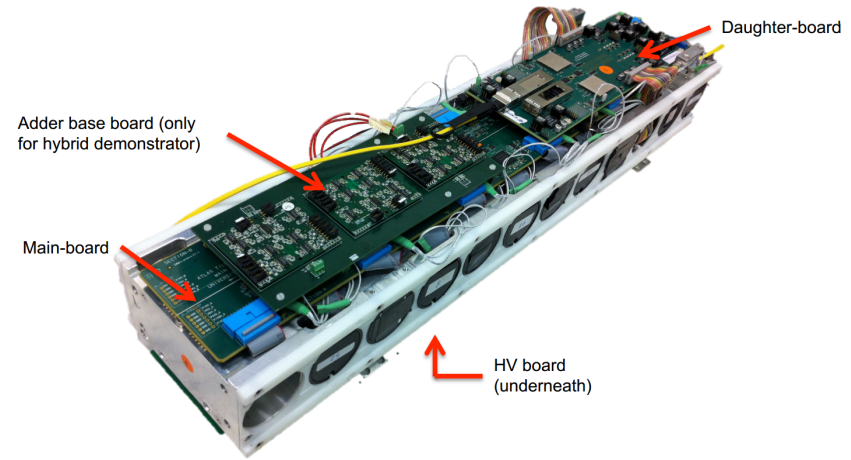

Fig. 1. Picture of a completely mounted mini-drawer, including main board, daughter board and HV-opto. Not visible in the picture are the 3-in-1 and the LVPS.

\section{SUMMARY}

To gain experience with the new design, a demonstrator project has been initiated, aimed at installing and running a prototype of the upgraded electronics in an ATLAS TileCal super-drawer. So as not to disrupt data taking, it must also be able to operate seamlessly with the present system. This means that the demonstrator still needs to provide analog trigger signals to the analog summation boards. The demonstrator on-detector electronics consist of 12 modified 3in1 front-end boards [3], a main board [4] for digitization, a daughter board for communication and control [5] and a programmable high voltage power supply (HV-opto) all mounted in a so called mini-drawer (a quarter of a standard superdrawer). To power the system dedicated $10 \mathrm{~V}$ power supplies (LVPS) were used [6]. The full mounted set-up is shown in figure 1. Due to space restrictions the 3-in-1 front-end boards are mounted directly onto the PMTs and therefore not visible. The LVPS is not shown because in the final system it will not be located next to the mini-drawer.

Since the demonstrator will be mounted in an area where access is not often possible, redundancy and fail safe operation have been important issues when designing the system. Components and thus whole boards could fail not

Corresponding author's e-mail: muschter@fysik.su.se.

S. Muschter, H. Aakerstedt and C. Bohm are with Fysikum, Stockholm University, 10691 Stockholm, Sweden

K. Anderson, M. Oreglia and F. Tang are with Enrico Fermi Institute, The University of Chicago, 5640 S. Ellis Ave, Chicago, IL 60637, USA.

G. Drage and A. Paramonov are Argonne National Laboratory, IL 60439 , 
only due to faulty components but also due to the radiationo environment in which the demonstrator is used. For this reason special care was taken during component selection, Components that are not classified as radiation hard were tested to ensure that they were suitable for operation within TileCal. Furthermore, it was attempted to minimize the number of components used to decrease the risk of failure. In case of component failure a built-in redundancy scheme will minimize the risk of losing data from an entire mini-drawer.

The current system implemented redundancy by reading the same scintillating block with two PMTs, processing thenaso in one digitizer board and sending the signals off-detecto ${ }^{11}$ using one fiber-connection. Power is distributed by one single ${ }_{33}^{132}$ LVPS per super-drawer, containing several bricks, serving as4 supplies for different voltages.

In the demonstrator the PMT duplication is consistent acros ${ }_{139}^{138}$ the whole system design, practically dividing the readouto chain into two independent halves. Every board is electricallyt divided in two, each half with its own local voltage regulation $\prod_{143}^{142}$ monitoring, control and readout electronics.

The power distribution network was adapted by powering $9_{6}^{145}$ each half independently with a supply voltage of $10 \mathrm{~V} \mathrm{de} 47$ livered by one brick, with the option of sharing power via a diode-or coupling if one power brick fails. Voltage regulation is performed locally on every board.

To communicate with the off-detector electronics, two QSFP+ modules are available, each powered by its corresponding half. Since only one will be used during normal operation it is able to communicate with both FPGAs at the same time. For enabling FPGA remote programming, each QSFP+ module has a custom made high speed de-serializer (GBTx [7]) connected to it, reserving one receiving channel for remote programming and external control. To increase redundancy in the same manner as before every GBTx is able to communicate with both sides of the daughter board and in particular with the Kintex 7 JTAG interface. This means that if one QSFP+ module fails, the off-detector electronics will still be able reprogram and thus recover both daughter board FPGAs. The benefit of making the JTAG interface directly accessible to the off-detector electronics is that this will improve the FPGA fail safe operation in a radiation environment as well. Utilizing Kintex 7 tools that allow scrubbing the configuration memory through the JTAG chain it is possible to correct multi bit errors in the FPGA without needing to stop and reprogram the FPGA.

To improve noise performance and linearity the 3in1 front-end boards were revised. Special care was taken to ensure a separation between the analog signal shaping and the digital signal sampling part of the on-detector system. As a result the ADC sampling width was increased from 10 bit to 12 bit. Thus the total dynamic range of the system has been slightly increased to 17 bits. Finally all critical transmission lines were simulated to improve data integrity along the whole system.

\section{CONCLUSION}

Currently four complete setups have been assembled and used for soft- and firmware development in order to improve stability and functionality. Noise and performance measurements were made in order to characterize the hardware. A full article with detailed descriptions about the demonstrator hardware and measurements has been submitted to the Transaction on Nuclear Science(TNS).

\section{REFERENCES}

[1] ATLAS Collaboration, ATLAS Tile Calorimeter Technical Design Report, CERN-LHCC 96-042, 1996

[2] ATLAS Collaboration, The ATLAS Experiment at the CERN Large Hadron Collider, in Journal of Instrumentation 3, no.08 (2008): S08003.

[3] F. Tang et al., Design of the front-end readout electronics for ATLAS tile calorimeter at the $s L H C$, Real Time Conference (RT), 2010 17th IEEENPSS, IEEE, 2010

[4] F. Tang et al., Upgrade analog readout and digitizing system for ATLAS TileCal demonstrator. in Proceedings of Nuclear Science Symposium and Medical Imaging Conference (NSS/MIC) IEEE, Oct. 27 - Nov. 02, 2013, Seoul, Korea

[5] S. Muschter et al., Development of a digital readout board for the ATLAS Tile Calorimeter upgrade demonstrator. in Journal of Instrumentation, 2014, 9. Jg., Nr. 01, S. C01001.

[6] G. Drake, Design of a new switching power supply for the ATLAS TileCAL front-end electronics. in Journal of Instrumentation, 2013, 8. Jg., Nr. 02, S. C02032.

[7] P. MOREIRA, et al. The GBT-SerDes ASIC prototype. in Journal of Instrumentation, 2010, 5. Jg., Nr. 11, S. C11022. 